\title{
LASER CLADDING BY POWDER INJECTION: OPTIMIZATION OF THE PROCESSING CONDITIONS
}

\author{
M.C. SAHOUR, A.B. VANNES* and J.M. PELLETIER \\ GEMPPM-CALFETMAT, Bât. 502, INSA, F-69621 Villeurbanne cedex, France \\ *MMP-CALFETMAT, BP. 163, F-69131 Ecully cedex, France
}

\begin{abstract}
An experimental study of the influence of laser procesing parameters on geometrical features of coatings is presented in this study. Different materials are used and a phenomenological interpretation of the results is proposed to explain the different phenomena, especially the occurence of a threshold value for the feed rate.
\end{abstract}

\section{I - Introduction :}

Nowadays, the realization of metallic coatings by laser processing, with $\mathrm{Co}$ or $\mathrm{Ni}$ base alloys, is effectively used in different industries, as automotive or aeronautic ones. Various substrates have been involved: low carbon steels, nickel or titanium base alloys.(1,2). However, parameters employed during this operation have a critical influence on the results, i.e. :

- soundness and absence of porosity in the surface layer,

- homogeneity,

- dilution of the addition element,

- size and features of the microstructure, as well as nature of the formed phases,

- hardness of the coating.

In the present study, we determine the influence of the following parameters : specific laser output power, scanning speed or interaction time and powder rate.

\section{II - Experimental procedure :}

The coating materials, Cenium 36 (Ce36), METCO 18C (M18C) and METCO 41C (M41C) alloy powders, which had the nominal compositions listed in table I, were sprayed with a METCO 4MP spray system, onto a grit blasted stainless steel substrate (304L).

$\begin{array}{lcccccccccc} & \mathrm{Co} & \mathrm{Cr} & \mathrm{Ni} & \mathrm{Fe} & \mathrm{C} & \mathrm{W} & \mathrm{Si} & \mathrm{Mn} & \mathrm{B} & \mathrm{Mo} \\ \text { M18C } & 40 & 18 & 26.8 & 2.5 & 0.2 & - & 3.5 & - & 3 & 6 \\ \text { M41C } & - & 17 & 12 & 67.4 & 0.1 & - & 1 & - & - & 6 \\ \text { Ce36 } & - & 30 & 36 & 20 & 1 & 5 & - & - & 3 & 9\end{array}$

table I : nominal compositions (wt\%) of the different powders.

Specimen were mounted on a numerically controlled $X-Y$ table and irradiated with a continuous $\mathrm{CO}_{2}$ laser, the power of which is up to $3.6 \mathrm{~kW}$. Laser beam is focused by a spherical $10^{\prime \prime} \mathrm{Zn}-\mathrm{Se}$ lens. Scanning rateV of the sample under the laser beam ranged from 3 to $15 \mathrm{~mm} / \mathrm{s}$. A variation of the interaction time, defined by $\tau=d / V_{T}$, where $d$ is the equivalent beam diameter on the sample (3) was thus possible. During scanning of the sample, argon was blown through a gas nozzle to prevent oxydation. No absorbing coating was used, in oder to avoid contamination of the sample during melting. 
For optical micrography and geometrical features determination, samples were cutted, polished and etched in the following reagent : $150 \mathrm{~cm}^{3} \mathrm{HCl}, 25 \mathrm{~g} \mathrm{~K}_{2} \mathrm{Cr}_{3} \mathrm{O}_{7}, 50 \mathrm{~cm}^{3} \mathrm{H}_{2} \mathrm{O}$.

\section{III - Experimental results :}

1 - Optimization of the laser processing conditions :

For a given system (substrate+powder), different parameters may be changed : laser output power $\mathrm{P}$, scanning rate $V_{T}$. But an other one is the defocusing distance $Z$, i.e. the distance between the focus point of the lens and the specimen. Fig. 1 shows for example the influence of this distance on the micrographic aspect of the coating $\left(P=1600 \mathrm{~W}, V_{T}=6.6 \mathrm{~mm} / \mathrm{s}\right.$, powder feed rate $\mathrm{F}=30 \mathrm{~g} / \mathrm{mn}$; powder Ce36; substrate $304 \mathrm{~L}$ ). A large defocusing distance induces only a very slight melting of the substrate and therefore dilution is very limited. This coating looks like a plasma sprayed one. However a detailed observation reveals the existence of a metallurgical bounding between the two materials and consequently adhesion is not too weak. A smaller defocusing (fig $1 \mathrm{~b}$ ) leads to a surface alloying : bounding is much better, but dilution is higher. So, depending on requirements, this processing parameter may be adapted. Further experiments are carried out with $\mathrm{Z}=-40 \mathrm{~mm}$ (focusing point above the sample). In such conditions beam diameter on the substrate is about $3 \mathrm{~mm}$.

Let us mention that no cracks or porosity are observed.

2 - influence of incident laser power and interaction time :

For given values of defocusing $Z$, powder feed rate $F$ and for a given powder, the effective section $S$ of the coating was measured by optical microscopy on a transversal cross-section of the sample. Results are plotted on fig. 2 and fig. 3 , and show that :

- for a given laser power, $S$ increases with $1 / \mathrm{V}_{\mathrm{T}}$, i.e. increases with interaction time $\tau=\mathrm{d} / \mathrm{V}_{\mathrm{T}}$.

- for a given scanning rate $V_{T}, S$ increases with $P$. However a threshold is observed; below a critical value, melting of the powder does not occur and therefore no coating is formed. The critical value $\mathrm{P}^{*}$ is the larger the smaller the interaction time.

Similar results are obtained with the other powders.

3 - Influence of the feed rate $F$ :

The influence of the feed rate on the cross-section surface is shown on fig.4, for three different powders. The three curves have the same aspect. It is clearly observed that that beyond a critical value $\mathrm{F}^{*}$, a sudden increase occurs. Interpretaion of this phenomenon could be as follows : as the powder rate increases, the screen of particles between the incoming laser beam and the substrate becomes more and more opaque and the global absorpion coefficient increases, by multiple scattering of the beam in the "powder cloud". A kind of "percolation threshold" is obtained (see further).

4 - parametrical representation :

The experimental results may be simply expressed by the following relation :

$$
\ln (S)=\ln (k)+\left(a V_{T}+b\right) \ln (P)
$$

where $k$, $a$ and $b$ are constants, depending on materials, granulometry and velocity range ( $v>$ or < $10 \mathrm{~mm} / \mathrm{s}$ ). These parameters could be related to thermopthysical properties of powders. However a correlation is difficult to establish, since the number of parameters is important : nature of materials, average particles size, distribution of the dimensions, features of the surface (presence of oxides, roughness...). So this correlation is very difficult to establish.

\section{IV - Discussion of the results :}

From experimental results, typical values of both interaction time and incident power density may be deduced. An analysis of the physical situation shows that different problems occur during laser cladding :

1 - energy to supply for melting :

A rough estimation gives :

$$
E=m\left(c \Delta \Theta+\Delta H_{m}\right)
$$

for the energy $\mathrm{E}$ required for melting a mass $\mathrm{m}$ of powder; $\mathrm{c}$ is the specific heat, $\Delta \Theta$ the temperature increase $\left(=T_{m}-T_{0}, T_{m}\right.$ : melting temperature, $T_{0}$ : initial temperature). $\Delta H_{m}$ : latent heat of melting. 
Powders used contain mainly $\mathrm{Co}, \mathrm{Ni}, \mathrm{Fe}$ and $\mathrm{Cr}$. Their thermophysical properties are very similar (4) and average values are as follows : $\rho=8 \mathrm{~g} / \mathrm{cm}^{3}, c=0.5 \mathrm{~J} / \mathrm{g}, \Delta \mathrm{H}_{\mathrm{m}}=300 \mathrm{~J} / \mathrm{g}$ and $\mathrm{T}_{\mathrm{m}}=1500^{\circ} \mathrm{C}$. Therefore for $\mathrm{m}=1 \mathrm{~g}$, the energy required for meiting is $\mathrm{E}=1 \mathrm{~kJ}$. So for a feed rate of $30 \mathrm{~g} / \mathrm{mn}(0.5 \mathrm{~g} / \mathrm{s})$, the absorbed irradiance required for melting this powder is $500 \mathrm{~W}$. The value of the corresponding laser beam power depends on the absorptivity of the particles. It is well known (4) that this coefficient $A$ is very small for metallic surfaces at $\lambda=10.6 \mu \mathrm{m}\left(\mathrm{CO}_{2}\right.$ laser wavelength). But for powder particles, two additives phenomena have to be taken into account :

- the existence of thin layers of surface oxides (always observed on small particles) increases the absorption coefficient. easier.

- A is much higher for liquids than for solids. As soon as melting is initiated, processing is much

- the powder constitutes a kind of cloud and so multiple scattering occurs in this medium. So a given particle will receive several times the laser beam and the effective absorption coefficient of this medium is much higher than a single surface one's and depends critically on both particle size and density. This dependence can explain the threshold observed in fig.4.

2- time required for melting:

For a given spherical particle, the time required for the homogeneization of the temperature is given, by : $\mathrm{t}^{*}$ $=r^{2} /(2 \alpha)$, where $r$ is the particle radius and $\alpha$ the thermal diffusivity (5). For metals, the diffusivity is typically $10^{-5} \mathrm{~m}^{2} / \mathrm{s}$. So for small powders $(\mathrm{r}<50 \mu \mathrm{m}), \mathrm{t}^{*}$ is smaller than $100 \mu \mathrm{s}$. Taking into account the processing parameters $\left(\tau, V_{\mathrm{T}}\right)$, it may be concluded that the real interaction time is always larger than $\mathrm{t}^{*}$ and therefore this stage of temperature homogeneization may be neglected.

\section{V - Conclusion :}

From our experimental results, the following conclusions may be deduced :

- with a given laser and a given spraying system, geometrical features and dilution of the coating may be easily changed, by modifying laser power, scanning rate or defocusing distance, section :

- parametric laws have been obtained for the effective surface of the coating, measured on a cross-

$$
\ln (S)=\ln (k)+\left(a V_{T}+b\right) \ln (P)
$$

where $k, a$ and $b$ are constants. A simple thermal description of this phenomenon gives a good estimation of the parameters,

- for high powder feed rates, the efficiency of the processing suddenly increases, due to the formation of a dense screen, which absorbs very efficiently the incoming laser beam.

\section{References :}

/1/ MAWELLA, K.J.A and HONEYCOMBE, R.W.K., J. Mat. Sci., 19 (1984) 3760.

/2/ SINGH, J. and MAZUMDER, J, Metall. Trans.A, 18A (1987) 313.

/3/ MERLIN, J. and DIETZ, J., Rev. Phys. Appl., 22 (1987) 735.

14/ TOULOUKIAN, Y.S. and DEWITT, D.P., "Thermophysical Properties of Matter", Plenum Press, N.Y. (1972).

15/ LAGAIN, P., Thesis, Aix-Marseille (1989), 250p. 

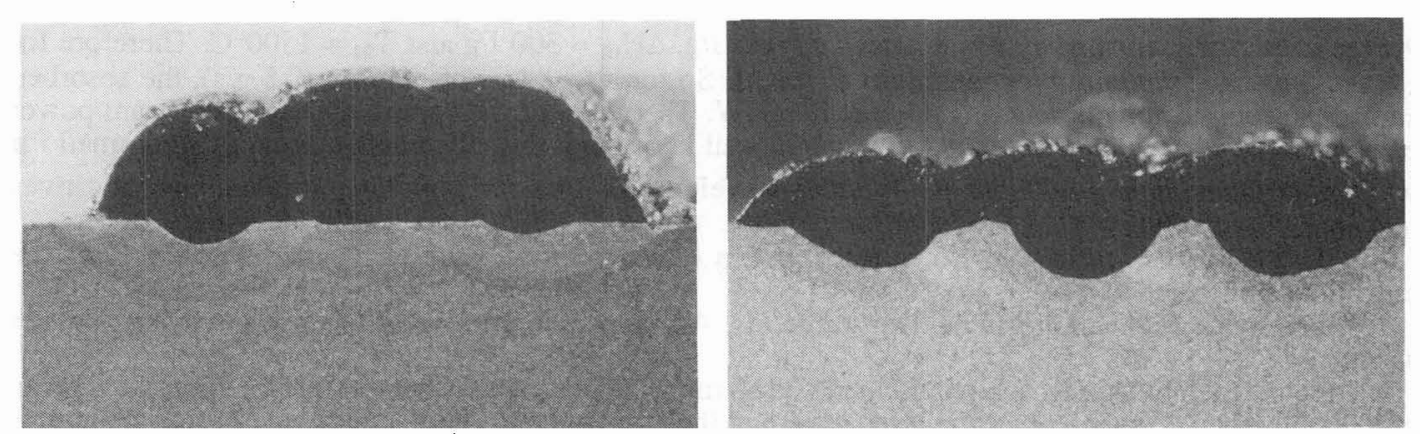

Fig. 1 : micrography of a coating (a) $(Z=-40$ $\mathrm{mm})$ and a surface alloy (b) ( $\mathrm{Z}=-20 \mathrm{~mm})$; powder : Metco 18C, substrate : 304L steel, $P$ $=1600 \mathrm{~W}, \mathrm{~V}_{\mathrm{T}}=6.6 \mathrm{~mm} / \mathrm{s}, \mathrm{F}=30 \mathrm{~g} / \mathrm{mn} .\left({ }^{*} 15\right)$
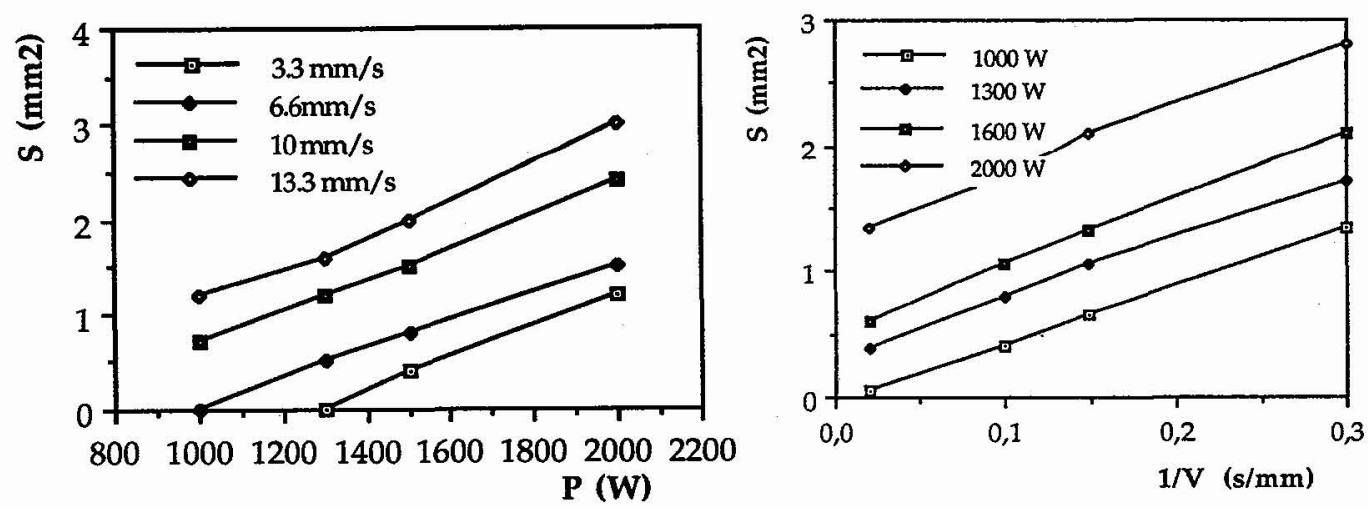

Fig. 2 : influence of the laser power on the effective surface of the coating ( Ce36, 304L, Z $=-40 \mathrm{~mm}, \mathrm{~F}=30 \mathrm{~g} / \mathrm{mn}$ ).

Fig. 3 : influence of the inverse of the scanning rate on the effective surface of the coating ( $\mathrm{Ce} 36,304 \mathrm{~L}, \mathrm{Z}=-40 \mathrm{~mm}, \mathrm{~F}=$ $30 \mathrm{~g} / \mathrm{mn}$ ).
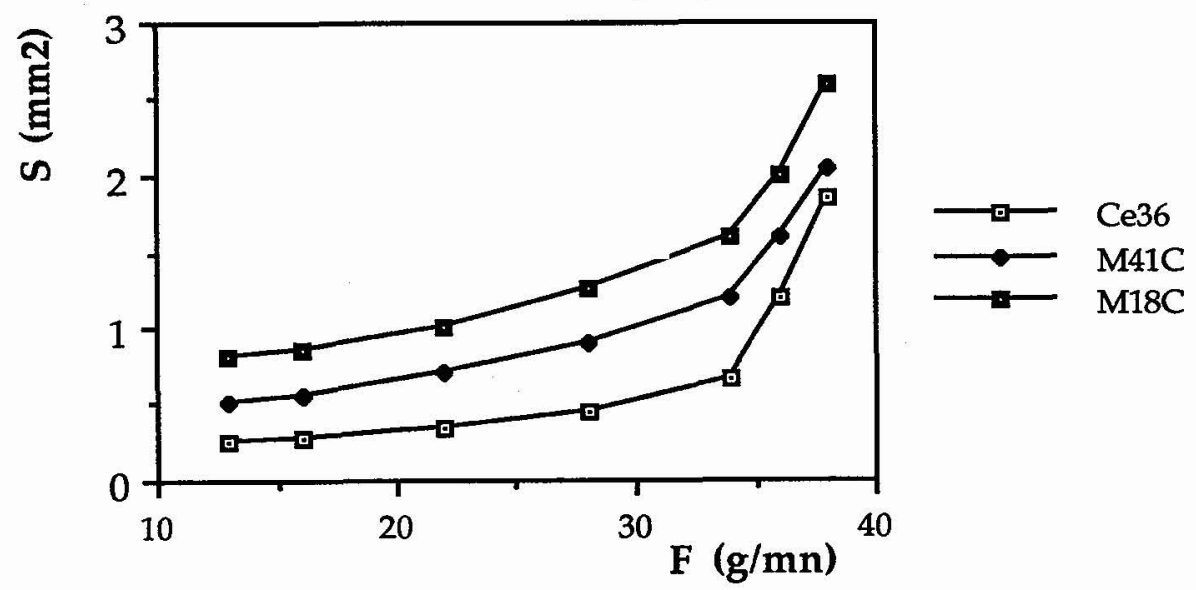

Fig. 4 : influence of the powder feed rate on the effective surface of the coating ( $\mathrm{Ce} 36$ or M18C or M41C, 304L, $Z=-40 \mathrm{~mm}, P=1500$ $\mathrm{W}, \mathrm{V}_{\mathrm{T}}=6.6 \mathrm{~mm} / \mathrm{s}$ ). 